\title{
Effective physical optics hands-on experience through the characterization of a CD and a DVD
}

\author{
A. Márquez, M. Álvarez, C. Neipp, D. Méndez, A. \\ Beléndez
}

A. Márquez, M. L. Álvarez, C. Neipp, D. Méndez, A. Beléndez, "Effective physical optics hands-on experience through the characterization of a CD and a DVD," Proc. SPIE 9664, Ninth International Topical Meeting on Education and Training in Optics and Photonics, 96642L (24 October 2005); doi: $10.1117 / 12.2207707$

SPIE Event: Ninth International Topical Meeting on Education and Training in Optics and Photonics, 2005, Marseille, France 


\title{
Ref ETOP047
}

\section{Effective physical optics hands-on experience through the characterization of a CD and a DVD}

\author{
A. Márquez, M.L. Álvarez, C. Neipp, D. Méndez and A. Beléndez
}

\begin{abstract}
We present a fruitful student's lab experiment allowing for an effective learning of some of the basics in physical optics. We proceed with the characterization of the surface structure of a compact disk (CD) and a digital versatile disk (DVD). This experiment is an effective means to have a direct hands-on experience on diffraction, Young's fringes interference phenomena, and diffraction chromatic dispersion. The fact that both CD and DVD are widespread consumer electronics devices enhances the interest of the student on the experiment.
\end{abstract}

1. Introduction

\section{Summary :}

Generally physical optics lab experiments involve the use of diffraction gratings to study the basics of diffraction phenomena and to analyse the grating law [1]. To introduce the basics on interference phenomena the two slits experiment is normally used, thus producing the Young's fringes [1] on a screen at a certain distance. Typical schemes to reproduce the two slits experiment are the Fresnel biprism or the Lloyd's mirror. These experiences allow for a direct verification by the student of the theory given in class. However, sometimes they may look very academic and not very appealing for the student. This is especially true in non Physics degrees. On the other hand, we can find some technological devices which may be as useful as the conventional diffraction gratings and the Fresnel biprism to provide a basic introduction to physical optics phenomena. One of them is the compact disk (CD), and an even more interesting device is the digital versatile disk (DVD) [2]. Several teaching experiments have been proposed in the literature using the $C D[3,4]$.

For some years we have run a CD and DVD based practice lab addressed to first year electrical engineers (Image and Sound specialty) and to first year computer engineers in the Polytechnic School of the University of Alicante. The physics subject in the first year of the degree is divided in theory classes, problem solving tutorials, and lab experiments. In the lab experiments we considered a good idea to use the CD and the DVD to introduce the basics on physical optics phenomena. We knew about the use of the CD to study the grating law [4]. Our first aim was to complete the experiment with the addition of the DVD. However, during the preparation of the experiment it was a nice surprise to find that the double layer structure of the DVD produced what looked as Young's fringes. We found that these Young's fringes allowed for the measurement of the distance between the two layers in the DVD ( $\cong 50 \mu \mathrm{m})$. In this way, we could use the CD and the DVD not only to study diffraction but also to study interference phenomena. The experiment also allowed for the introduction of some basics on optical storage of information. An interesting point of the experiment is that the students learn how the theory given in class can be effectively used to model a physics phenomenon such as the interference fringes in the DVD. All together has proven a very appealing lab experiment for the students. In the next Section we give the outline of the practice with some theory. Eventually, we present the main conclusions. 
2. lab experiment theory and outline

The goals in the lab experiment are to determine the separation between the tracks in the CD and in the DVD, to determine the separation between the two layers in the DVD, to calculate the storage capacity in both optical disk formats, and some qualitative analysis. In order to develop the experiments the material that we use is: an optics breadboard, a He-Ne laser, white-light lamp (e.g. the illumination light bulbs in the lab), mirror, diffusing screen, rule, a CD, a DVD, posts and mounting posts.

Next we introduce some basic theory dealing with the structure of the CD and DVD. An optical disk (CD or DVD) is basically a reflective surface in which a series of marks (pits) are registered to codify the information. The pits are distributed along a spiral line (the track) between the outer and the inner radius of the disk. We can consider the spiral line as composed of a series of concentric rings with a separation of $\Lambda$ (the track pitch). Depending on the reading-write format of the disk (read-only, writable, rewritable) the physical characteristic of the pits is different (depth modulation, reflectance modulation), but in any case there is a spiral track line grooved onto the disk which allows for the correct tracking of the spinning disk by the reading-writing pickup unit. If we make a cut along a radius of the disk we get a periodic groove structure, which can actually be considered as a reflective diffraction grating with a period $\wedge(1.6 \mu \mathrm{m}$ for the CD and $0.74 \mu \mathrm{m}$ for the DVD). If we illuminate the disk with an unexpanded laser beam with a wavelength $\lambda$ we clearly obtain the orders of diffraction, whose directions are given by the grating law,

$\sin \theta_{m}=\sin \theta_{i}+m \frac{\lambda}{\Lambda}$

where $\theta_{m}$ and $\theta_{i}$ are the angles with respect to the normal of the disk surface for the diffraction order $\mathrm{m}$, and for the incidence beam. When we know the period $\Lambda$ and the inner $R_{\text {in }}$ and outer $R_{\text {out }}$ radius of the spiral track we can calculate the length $L$ of the track.

$L=\pi\left(R_{\text {out }}^{2}-R_{\text {in }}^{2}\right) / \Lambda$

For a given average bit density along the track ( $1 \mu \mathrm{m}$ for a CD and $0.3 \mu \mathrm{m}$ for a DVD) we can calculate the storage capacity for each of the optical disks.

In the case of the DVD we have a double layer structure at each face of the disk with the corresponding grooved spiral track at each layer. When we illuminate the surface of the DVD we obtain the reflective diffraction orders given by each of the two layers at the illuminated face. We find that each of the diffraction orders have an inner structure composed of interference fringes.

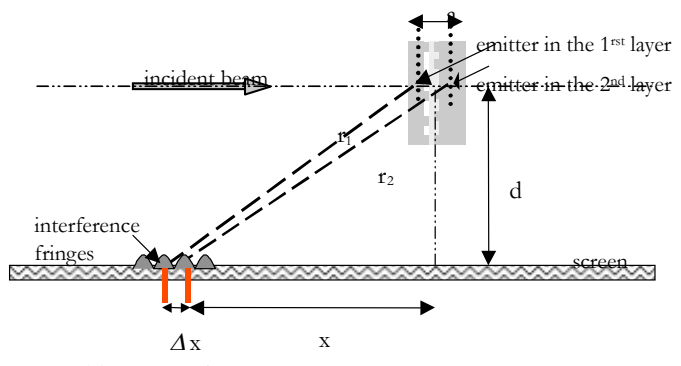

Fig.1. Two Young's slits model for the two emitters in the DVD layers. Considering an easy model (see Fig. 1) we can obtain the separation a of the layers of the DVD by assuming that the point where the incident laser impact on each of the two layers behaves as a light emitter. The two emitters can be considered as the two slits in the Young's fringes experiment. One noticeable difference with the conventional case of the two slits is that the two emitters in the DVD only emit at discrete angles $\theta_{m}$. Due to the 
small period $\Lambda$ in the DVD the angle of diffraction $\theta_{m}$ is out of the paraxial regime and we have to calculate a more rigorous expression for the separation between the fringes $\Delta x$ produced on an observation screen placed at a distance $d$ from two slit-like emitters, given by,

$\Delta x=\lambda \frac{\sqrt{x^{2}+d^{2}}}{a}$

In the paraxial regime, $x<<d$, the previous expression simplifies into the usual Young's interference equation.

After the basic theory has been introduced we outline the measurements that are performed in the lab. On one hand, we ask the students for some quantitative evaluations, and on the other hand we ask them for some qualitative analysis. The quantitative evaluations deal with the calculation of the track separation $\Lambda$, the total length $L$ of the track, the storage capacity of the disk, and the separation a of the layers (in the DVD). For these evaluations the students have to measure the deflection angle $\theta_{m}$, the inner $R_{\text {in }}$ and outer $R_{\text {out }}$ radius, and the distances $\mathrm{d}, \mathrm{x}$ and $\Delta x$ (as given in Fig. 1 for the DVD). The students have to evaluate the parameters together with its associated uncertainty. Further qualitative analysis proposed to the students are to connect the orientation of the diffraction orders with the orientation of the tracks on the disk (we can consider the disk as a reflection grating with rotational symmetry), and to discuss why when illuminated with ambient light we observe the color decomposition of the visible spectrum (chromatic dispersion due to diffraction).

\section{Conclusions}

The use of widespread technological devices, such as the CD and the DVD, is usually more appealing to students than the more academic diffraction gratings or the Fresnel biprism. We have exposed some possibilities to present the basics on diffraction and interference in an optical physics lab using a CD and a DVD.

\section{Acknowledgments}

This work was supported by Generalitat Valenciana, Spain (projects GV01-130, GV04A/574 and GV04A/565).

\section{References}

1. E. Hecht, Optics, Addison Wesley, 4th edition (2001).

2. Optical Storage Technology Association. http://www.osta.org

3. P.M. Lane, "Compact disc players in the laboratory: Experiments in optical storage, error correction, and optical fiber communication", IEEE Trans. on Education. 44(1), 47-60 (2001).

4. J.E. Kettler, "The compact disc as a diffraction grating", Am. J. Phys. 59(4), 367-368 (1991). 